\title{
A giant radio halo in the low luminosity X-ray cluster Abell 523
}

\author{
G. Giovannini ${ }^{1,2}$, L. Feretti ${ }^{2}$, M. Girardi ${ }^{3}$, F. Govoni ${ }^{4}$, M. Murgia ${ }^{4}$, V. Vacca ${ }^{4,5}$, and J. Bagchi ${ }^{6}$ \\ 1 Dipartimento di Astronomia, via Ranzani 1, 40127 Bologna, Italy \\ e-mail: ggiovann@ira.inaf.it \\ 2 Istituto di Radioastronomia-INAF, via P.Gobetti 101, 40129 Bologna, Italy \\ 3 Dipartimento di Fisica-Sezione di Astronomia, via Tiepolo 11, 34143 Trieste, Italy \\ ${ }^{4}$ Osservatorio Astronomico di Cagliari-INAF, Strada 54, Loc. Poggio dei Pini, 09012 Capoterra (Ca), Italy \\ 5 Dipartimento di Fisica, Università degli studi di Cagliari, Cittadella Universitaria, 09042 Monserrato (CA), Italy \\ ${ }^{6}$ Inter University Center for Astronomy and Astrophysics (IUCAA), Post Bag 4, Ganeshkhind, Pune 411 007, India
}

Received 21 March 2011 / Accepted 14 April 2011

\section{ABSTRACT}

\begin{abstract}
Context. Radio halos are extended and diffuse non-thermal radio sources present at the cluster center, not obviously associated with any individual galaxy. A strong correlation has been found between the cluster X-ray luminosity and the halo radio power. Aims. We observe and analyze the diffuse radio emission present in the complex merging structure Abell 523, classified as a low luminosity X-ray cluster, to discuss its properties in the context of the halo total radio power versus X-ray luminosity correlation. Methods. We reduced VLA archive observations at $1.4 \mathrm{GHz}$ to derive a deep radio image of the diffuse emission, and compared radio, optical, and X-ray data.

Results. Low-resolution VLA images detect a giant radio halo associated with a complex merging region. The properties of this new halo agree with those of radio halos in general discussed in the literature, but its radio power is about a factor of ten higher than expected on the basis of the cluster X-ray luminosity.

Conclusions. Our study of this giant radio source demonstrates that radio halos can also be present in clusters with a low X-ray luminosity. Only a few similar cases have so far been found. This result suggests that this source represents a new class of objects, that cannot be explained by classical radio halo models. We suggest that the particle reacceleration related to merging processes is very efficient and/or the X-ray luminosity is not a good indicator of the past merging activity of a cluster.
\end{abstract}

Key words. galaxies: clusters: general - galaxies: clusters: individual: Abell 523 - large-scale structure of the Universe

\section{Introduction}

Clusters of galaxies are characterized by X-ray emission from a hot intra-cluster medium (ICM, $T \sim 2-10 \mathrm{keV}$ ). Thermal emission is a common property of all clusters of galaxies and has been detected even in poor groups as well as in optical filaments connecting rich clusters. The ICM is believed to be shock heated by merging during the structure formation.

Diffuse non-thermal radio sources with steep spectra have been detected in the central regions of a large number of clusters of galaxies (see e.g. Giovannini et al. 2009; Venturi et al. 2008, and refs therein and review papers such as Feretti 2005; and Ferrari et al. 2008). They are giant radio sources with a spatial extent similar to that of the hot ICM, which are called radio halos. In addition, diffuse radio sources classified as radio relics are detected in cluster peripheral regions (see e.g. Giovannini \& Feretti 2004; and van Weeren et al. 2011). These sources are not directly associated with the activity of individual galaxies and are related to physical properties of the whole cluster. There is a substantial evidence that radio halos are found in clusters with significant substructures in X-ray images and complex gas temperature distributions, both signatures of cluster mergers (e.g. Feretti 1999; Govoni et al. 2004; Giovannini et al. 2009; Cassano et al. 2010). Classical radio halos show a centrally located and regular radio morphology. However, in several cases the radio halos are irregular and elongated as in A209, A401, and A2034.
A few radio halos such as A851 and A2218 are highly asymmetric, being located mostly to one side with respect to the cluster center. Giovannini et al. (2009) interpreted these structures as possibly reflecting the geometry of the merger.

Feretti (2000) discussed that the radio power of halos at $1.4 \mathrm{GHz}$ increases with the X-ray luminosity of the parent cluster, implying a direct connection between the radio and X-ray plasmas. Since the cluster X-ray luminosity and mass are correlated, it follows that radio halo power correlates with cluster mass (Feretti 2000; Govoni et al. 2001). The strong correlation between the radio halo total power at $1.4 \mathrm{GHz}$ and the X-ray cluster luminosity was recently discussed using higher quality statistics by Brunetti et al. (2007) and Giovannini et al. (2009), who show that the correlation is also present for low power radio halos $\left(P_{1.4}<10^{24} \mathrm{~W} / \mathrm{Hz}\right.$ and $\left.L_{x} \sim 10^{44} \mathrm{erg} / \mathrm{s}\right)$.

Giovannini et al. (2009) were the first to discuss whether a radio halo was present in A1213, an under-luminous X-ray cluster i.e. well outside the above correlation. A similar case was found in the cluster $0217+70$ by Brown et al. (2011). We do not consider radio relics because present models connect radio halos to turbulence in the ICM, while relic sources are correlated to the presence of shock waves. Brown et al. (2011) discussed whether the low X-ray luminosity in $0217+70$ could be due to an underestimate of the X-ray luminosity because of the hydrogen column density, which might be patchy at these low Galactic latitudes. Therefore, further X-ray observations are required to measure the absorption directly. We note that 


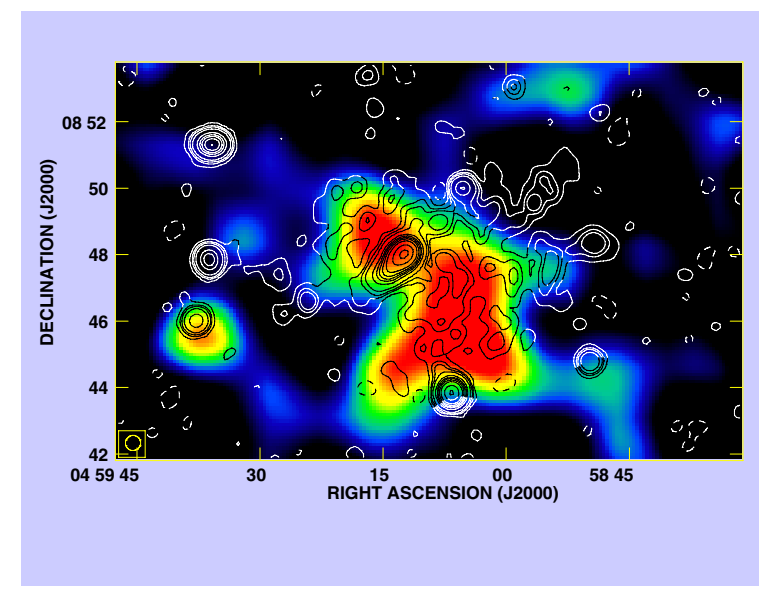

Fig. 1. High resolution radio image of A523 at $1.4 \mathrm{GHz}$ (contours). The HPBW is $25.5^{\prime \prime} \times 27.1^{\prime \prime}$ in PA $-55^{\circ}$. The noise level is $0.034 \mathrm{mJy} / \mathrm{beam}$; contours are: $-0.1,0.1,0.3,0.5,1,3,5,7,10,15,30$, and $50 \mathrm{mJy} / \mathrm{beam}$. Colors refer to the X-ray emission detected from the Rosat satellite.

Giovannini et al. (2009) discussed the peculiarity of the diffuse emission in A1213.

We present new VLA images from archive data of diffuse radio emission, found by chance by inspecting NVSS images, in A523, one more radio halo in a low X-ray luminosity cluster. We present in Sect. 2 the radio data and the X-ray and optical information. Discussion and conclusions are given in Sect. 3.

The intrinsic parameters quoted in this paper are computed for a $\Lambda$ CDM cosmology with $H_{0}=71 \mathrm{~km} \mathrm{~s}^{-1} \mathrm{Mpc}^{-1}, \Omega_{\mathrm{m}}=$ 0.27 , and $\Omega_{\Lambda}=0.73$. At $z=0.10$, the luminosity distance is $455 \mathrm{Mpc}$, and the angular conversion factor is $1.82 \mathrm{kpc} /$ arcsec.

\section{Radio images}

A523 was observed at $1.4 \mathrm{GHz}$ in line mode in 2005, with the VLA in the $\mathrm{C}(5 \mathrm{~h})$ and $\mathrm{D}(2 \mathrm{~h})$ configuration (project AB1180). Calibration and imaging were performed with the NRAO Astronomical Image Processing System (AIPS). After the editing of bad points, self-calibration was applied to both datasets, to remove residual phase and gain variations.

In Fig. 1, we show the final image obtained with $\mathrm{C}$ configuration data and natural weights (contours), superimposed on the X-ray image (colors) obtained with the Rosat satellite. The diffuse emission is also visible at this relatively high angular resolution and discrete sources are well separated. To obtain a deeper image of the diffuse source, we performed a full resolution (using uniform weighting) image with $\mathrm{C}$ array data, cutting out in addition the shortest baselines where some flux density from the extended halo was present. In this image, only discrete sources are present, and we used it to select the clean components of sources in the halo region with the CCEDT task. These components were subtracted from the D array uv-data, and we used resulting data to produce a low resolution image of the diffuse component (see Fig. 2). We used this image to measure the halo flux density and size. The main problems in this procedure are the following: 1) The presence of extended emission from a head-tail source located near the center of the radio halo. We compared the flux density of this source with the clean components used to subtract it from D-array UV data. We are confident to have subtracted more than $95 \%$ of the flux density of this extended tail source. 2) The elongated halo extension in the extreme east region. In this position, two discrete sources are present, one coincident with a X-ray source. However, the resid-

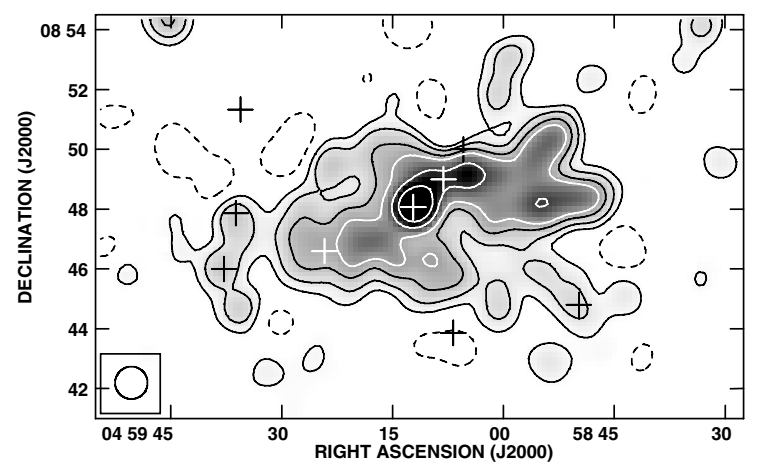

Fig. 2. Radio image of the halo in A523 (contours and gray scale) after subtraction of discrete sources. The HPBW is $65^{\prime \prime}$. The noise level is $0.04 \mathrm{mJy} / \mathrm{beam}$. Contours are: $-0.2,0.2,0.5,1,1.5,3,5 \mathrm{mJy} / \mathrm{beam}$. Crosses indicate the peak position of subtracted sources.

ual flux density in the low resolution image is too high to be interpreted as the residual of the subtracted sources. In any case, including this region or not will change the halo flux density by only $\sim 2.7 \mathrm{mJy}$.

The estimated halo flux density is $59 \pm 5 \mathrm{mJy}$, where the error includes the uncertainties in the source subtraction), corresponding to a total radio power $=1.47 \times 10^{24} \mathrm{~W} / \mathrm{Hz}(\log P=$ 24.17). The morphology is irregular with a largest angular size of $\sim 12$ arcmin in the EW direction, corresponding to $\sim 1.3 \mathrm{Mpc}$.

The radio emission detected at high resolution and shown in Fig. 1 permeates both merging clumps, as found e.g. in the Bullet cluster (Liang et al. 2000). This is also similar to the case e.g. of A2255 (Govoni et al. 2005) or A665 (Giovannini \& Feretti 2000) where the radio halos are elongated in the direction of the cluster merger. The radio structure in A523 is therefore typical of radio halos. When degraded to lower resolution, owing to the improved signal-to-noise ratio, the low brightness radio emission is more extended in particular in the E-W direction. The elongated structure does not show any morphological feature typical of radio relics such as high brightness filamentary structures or a transverse flux asymmetry (see e.g. van Weeren et al. 2011). We conclude that its classification as a radio relic is very unlikely. We also note that a fainter X-ray emission, not detected by ROSAT, could be present. We therefore classify the diffuse radio emission in A523 as a giant radio halo. The size and radio power of the halo are in good agreement with the correlation presented for radio halos by Giovannini et al. (2009).

\subsection{Optical and $X$-ray data}

Optically, A523 is a very rich cluster of Abell richness class $=2$ (Abell et al. 1989). It was classified to be of the RoodSastry morphological type "L", i.e. to have a linear configuration of galaxies (Struble \& Rood 1987). To date, only the redshift for the brightest cluster galaxy [BCG at RA $=04^{\mathrm{h}} 59^{\mathrm{m}} 12.97$, Dec $\left.=+08^{\circ} 49^{\prime} 41.3^{\prime \prime} \quad(\mathrm{J} 2000.0)\right]$ has been acquired $(z=0.1036$; Crawford et al. 1999). No radio emission is clearly associated with this galaxy. The visual inspection of DSS2-red image shows the presence of two clumps. To quantify this impression, we compiled a photometric catalog in a large region around the BCG $\left(\sim 30^{\prime}\right)$ from the SuperCOSMOS Sky Surveys $\left(\mathrm{SSS}^{1}\right)$ extracting the objects classified as "galaxies" that have both $B_{j}$ and $R$ magnitudes available. In this photometric catalog, we selected likely cluster members on the basis of the color-magnitude re-

${ }^{1}$ http://www-wfau.roe.ac.uk/sss/index.html 


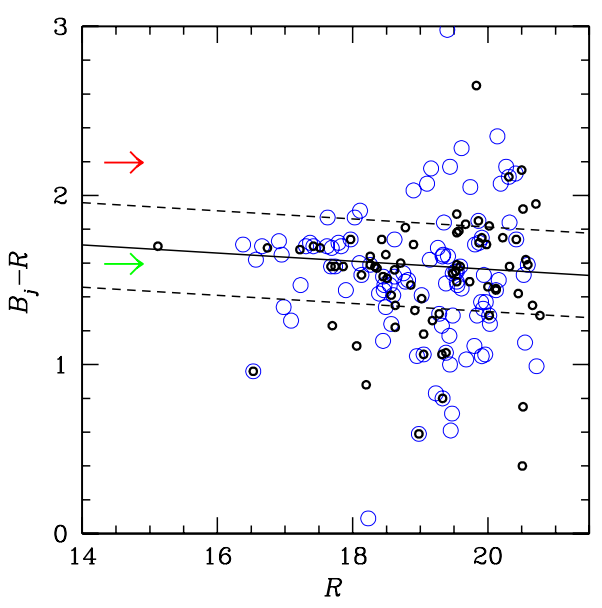

Fig. 3. $B_{j}-R$ vs. $R$ diagram for galaxies extracted from the SSS. Small circles indicate galaxies within a radius of $4^{\prime}$ from the BCG and used to fit the CMR (solid line). The dashed lines are drawn at \pm 0.25 mag from the CMR to delimitate the region of "likely cluster members". Large blue circles indicate galaxies within a radius of $4^{\prime}$ from the SSW peak. The green and red arrows roughly indicate predicted colors for earlytype galaxies at $z=0$ and $z=0.2$, respectively.

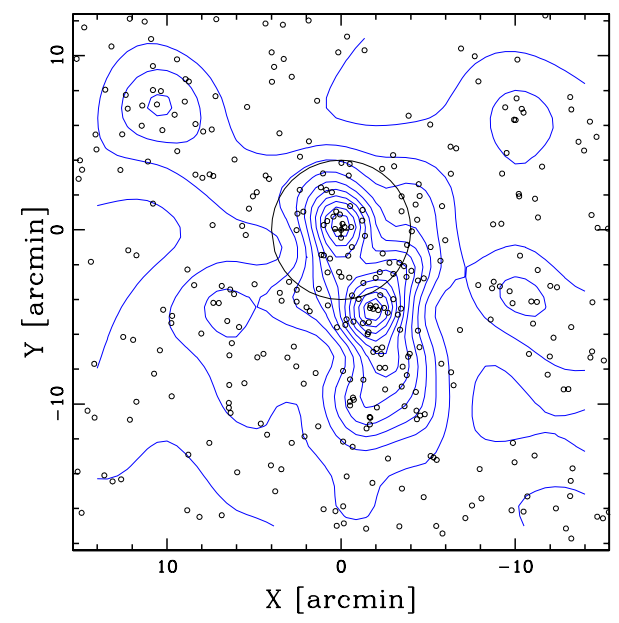

Fig. 4. Spatial distribution on the sky and relative isodensity contour map of likely cluster members with $r^{\prime} \leq 20$, obtained with the $2 \mathrm{D}$ DEDICA method. The large circle indicates the region of $4^{\prime}$-radius around the BCG used to fix the CMR.

lation (hereafter CMR), which indicates the early-type galaxy locus. To determine the CMR, we considered the 75 galaxies within a radius of $4^{\prime}$ from the BCG and applied a two-sigmaclipping fitting procedure, obtaining $B_{j}-R=2.044-0.024 \times R$ for a subsample of 54 galaxies. The color-magnitude diagram is shown in Fig. 3. From the photometric catalog, we considered as "likely cluster members" those galaxies lying within 0.25 mag of the CMR. Figure 4 shows a zoomed region of the contour map for the likely members ( 734 galaxies within the whole $\sim 30^{\prime}$ region). The galaxy distribution reveals two significant peaks $\sim 5^{\prime} \quad(\sim 0.5 \mathrm{Mpc})$ far: the NNE peak, coincident with the BCG, and the SSW peak, with no dominant galaxy, at $\mathrm{RA}=04^{\mathrm{h}} 59^{\mathrm{m}} 05^{\mathrm{s}} .4$, Dec $=+08^{\circ} 45^{\prime} 06^{\prime \prime} \quad(\mathrm{J} 2000.0)$. The SSW peak has the higher density and a larger population - by factors of 1.2 and 2.7 according to the $2 \mathrm{D}$ adaptive - kernel method (2D DEDICA, Pisani 1996). Figure 3 also shows the 105 galaxies of the photometric sample within a radius of $4^{\prime}$ from the SSW

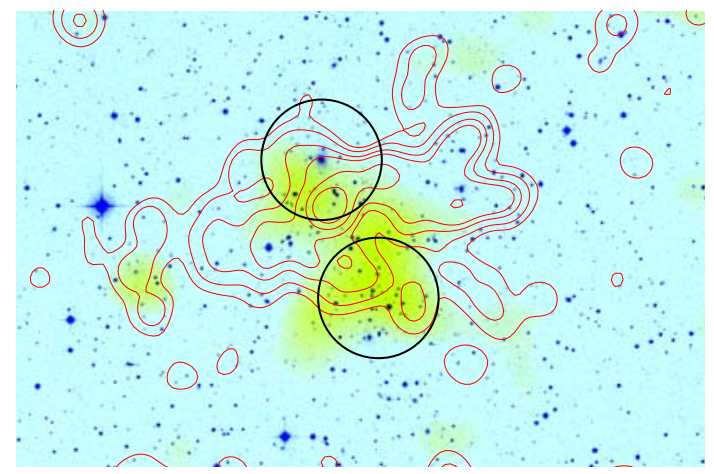

Fig. 5. Multiwavelength picture of A523. A smoothed Rosat image (green color) is superimposed on the DSS2-red image. The black circles indicate the regions of $2^{\prime}$-radius around the NNE and SSW galaxy groups. The BCG is clearly visible at the center of the NNE circle. Contour levels of the radio image of the extended halo after the subtraction of discrete sources are shown in red.

peak, whose properties can be accurately described by the above CMR, too, suggesting that both clumps are at a similar redshift.

Ebeling et al. (1998) report a cluster X-ray luminosity in the $0.1-2.4 \mathrm{keV}$ band of $1.07 \times 10^{44} \mathrm{erg} / \mathrm{s}$ (after cosmological corrections). Böhringer et al. (2000) report a slightly lower value of $0.9 \times 10^{44} \mathrm{erg} / \mathrm{s}$. The hot ICM (see Fig. 1) is clearly bimodal in agreement with the optical galaxy distribution. However, a clear shift between the galaxies and gas distribution is present (see Fig. 5) and the BCG is not coincident with the X-ray peak. The SSW structure is more extended and shows an irregular shape. The NNE clump is more compact and regular. The bright source, visible in the X-ray map at RA $=04^{\mathrm{h}} 59^{\mathrm{m}} 37 \mathrm{~s} .7$, Dec $=+08^{\circ} 45^{\prime} 50^{\prime \prime} \quad(\mathrm{J} 2000.0)$ is identified with a discrete unrelated radio source.

We conclude that optical and X-ray data that we have presented indicate a merging cluster structure, where the main cluster is identified with the SSW structure. This cluster as shown by its irregular shape and lack of a dominant galaxy, was not in a relaxed stage, and is now strongly interacting (major merging) with a more compact cluster (the NNE structure) dominated by a bright BCG.

\section{Discussion}

We present in Fig. 6 the plot of the total radio power and X-ray luminosity for radio halos as shown by Giovannini et al. (2009), where we have included $0217+70$ and A523. The dots refer to classical powerful radio halos in X-ray luminous clusters, which have been found to show a correlation between the radio power and the X-ray luminosity. The red triangles are outliers, i.e. they refer to the few known radio halos that are overluminous in radio with respect to the empirical radio - X-ray correlation.

We focus here on the objects that are overluminous in radio with respect to the empirical radio - X-ray correlation. We note that there might be some uncertainties in the computation of their parameters. In particular, A1213 displays a peculiar diffuse emission, and its size and radio power is lower than that of classical giant radio halos. Even if Giovannini et al. (2009) showed that small-size radio halos have the same properties as giant radio halos, data of higher statistical quality are necessary to confirm this result. In addition, the cluster $0217+70$, as discussed by Brown et al. (2011), is on the galactic plane therefore the X-ray luminosity could be affected by an unusually high absorption. On the other hand, the uncertainties in the determi- 


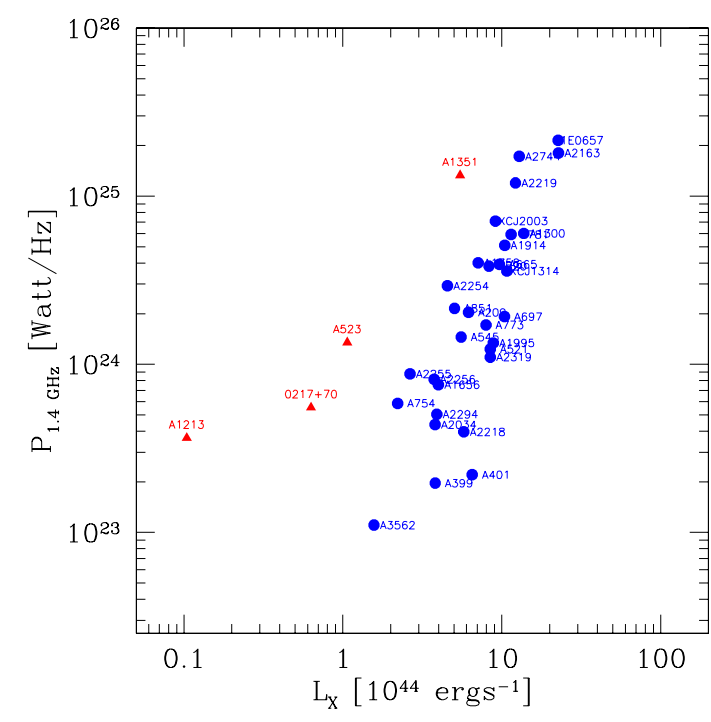

Fig. 6. Cluster X-ray luminosity between 0.1 and $2.4 \mathrm{keV}$ versus the total halo radio power at $1.4 \mathrm{GHz}$. Red triangles are outlier clusters discussed in the text. Blue dots are classical clusters from Giovannini et al. (2009).

nation of the radio power and the X-ray luminosity in the cluster A1351 (Giovannini et al. 2009) are smaller than the deviations from the correlation (however see Giacintucci et al. 2009).

In the case of A523, we are aware that the redshift is not well known, since only the redshift of the BCG is available. However, to be consistent with the correlation between the radio power and the X-ray luminosity, the clusters should be at a redshift of $\sim 0.2$. Figure 3 shows the predicted values of the $B_{j-}$ $R=1.4$ and 2.0 for the predicted early-type galaxies at $z=0.0$ and $z=0.2$, respectively (see Fig. 1 of Brown et al. 2000 and refs. therein) after a 0.2 mag decorrection for the Galactic absorption. Although these predictions cannot be used to provide a redshift estimate, they are useful to show to the reader the difference expected in the color-magnitude relation for a $z \sim 0.2$ cluster, thus strongly favouring the location of A523 at much lower distance. Moreover, at $z=0.2$, the halo size would be $\sim 2.4 \mathrm{Mpc}$, the largest radio halo known to date (see Giovannini et al. 2009).

The radio halo in A523 therefore represents a robust case of a giant radio halo clearly identified with an X-ray underluminous cluster. As discussed in Sect. 2, the X-ray and optical distribution is similar (bimodal) but not in perfect agreement. The thermal gas distribution is shifted with respect to the galaxy distribution; this is more evident for the smaller and more compact NNE clump, where the position of the BCG is not at the center of the gas distribution. A higher quality X-ray image is necessary to investigate whether the peculiarity of A523 could be related to the possibility that the compact NNE group just crossed the SSW cluster as in the case of the Bullet cluster (1E0657-56, Markevitch et al. 2002), and this strong and recent interaction affected in a different way the gas and galaxy distribution.

We conclude that there is firm observational evidence that giant radio halos can be associated with low luminosity X-ray clusters. These halos are overluminous in radio by at least an order of magnitude with respect to that expected from the extrapolation of the observed radio power - X-ray luminosity.

The radio - X-ray correlation is consistent with reacceleration models, which have been extensively discussed in the literature (Cassano 2010 and refs therein) and supported by the observations (e.g. Giovannini et al. 2009). In the framework of these models, the radio emitting particles in cluster radio halos gain energy from merger-driven turbulence in the ICM. Turbulence development on timescales of the order of $1 \mathrm{Gyr}$ is necessary to reaccelerate electrons to the energies needed to emit the observed synchrotron radiation at $\mathrm{GHz}$ frequencies, thus giant radio halos are expected to be strictly connected to massive systems that have undergone strong merging processes. In the framework of turbulent reacceleration models, Cassano 2010 suggests that very steep spectrum radio halos should exist that are detectable only at low frequencies, and be less luminous than predicted by the radio - X-ray correlation. The radio halo in A523, and a few similar objects, are instead more luminous in radio than predicted by the radio - X-ray correlation. These powerful radio halos, associated with clusters of low X-ray luminosity, do not appear to be described well by current models, hence are likely to represent a new class of objects, raising new questions about the origin of radio halos. They could be either young halos or clusters at a special time of the merger event, when particle acceleration processes have a higher efficiency (see Brunetti \& Lazarian 2011). Another possibility is that the X-ray luminosity might not be in these cases a good indicator of the previous cluster merging activity.

We note that the non-thermal diffuse radio emission in A523 does not follow the optical and the X-ray emission, unlike most other radio halos. The brighter halo region visible at high resolution (Fig. 1) is in good agreement with the X-ray bimodal distribution, but the more extended low brightness halo emission, which is most clearly imaged at low resolution, is elongated in the E-W direction i.e. in the direction perpendicular to the merger. This morphology could be related to peculiar cluster conditions that give rise to these overluminous radio halos and needs to be studied in better detail with more sensitive X-ray and radio data and radio spectral information.

Acknowledgements. The National Radio Astronomy Observatory is operated by Associated Universities, Inc., under cooperative agreement with the National Science Foundation.

\section{References}

Abell, G. O., Corwin, H. G. Jr., \& Olowin, R. P. 1989, ApJS, 70, 1 Böhringer, H., Voges, W., Huchra, J. P., et al. 2000, ApJS, 129, 435 Brown, M. J. I., Webster, R. L., \& Boyle, B. J. 2000, MNRAS, 317, 782 Brown, S., Duesterhoeft, J., \& Rudnick, L. 2011, ApJ, 727, L25 Brunetti, G., \& Lazarian, A. 2011 MNRAS, 412, 817

Brunetti, G., Venturi, T., Dallacasa, D., et al. 2007, ApJ, 670, L5 Cassano, R., 2010, A\&A, 517, 10

Cassano, R., Ettori, S., Giacintucci, S., et al. 2010, ApJ, 721, 82

Crawford, C. S., Allen, S. W., Ebeling, H., et al. 1999, MNRAS, 306, 857 Ebeling, H., Edge, A. C., Böhringer, H., et al. 1998, MNRAS, 301, 881 Ferrari, C., Govoni, F., Schindler, S., et al. 2008, Space Sci. Rev., 134, 93 Feretti, L. 1999, Diffuse Thermal and Relativistic Plasma in Galaxy Clusters, ed. H. Böhringer, L. Feretti, P. Schuecker, Max-Planck-Institut fur Extraterrestrische Physik, 3

Feretti, L. 2000, Invited review at the IAU 199 [arXiv:astro-ph/0006379] Feretti, L. 2005, Adv. Space Res., 36, 729

Giacintucci, S., Venturi, T., Cassano, R., et al. 2009, ApJ, 704, L54

Giovannini, G., \& Feretti, L. 2000, New Astron., 5, 335

Giovannini, G., \& Feretti, L. 2004, J. Korean Astron. Soc., 37, 323

Giovannini, G., Bonafede, A., Feretti, L., et al. 2009, A\&A, 507, 1257

Govoni, F., Feretti, L., Giovannini, G., et al. 2001, A\&A, 376, 803

Govoni, F., Markevitch, M., Vikhlinin, A., et al. 2004, ApJ, 605, 695

Govoni, F., Murgia, M., Feretti, L., et al. 2005, A\&A, 430, L5

Liang, H., Hunstead, R. W., Birkinshaw, M., \& Andreani, P. 2000, ApJ, 686, 701

Markevitch, M., Gonzalez, A. H., David, L., et al. 2002, ApJ, 567, L27

Pisani, A. 1996, MNRAS, 278, 697

Struble, M. F. \& Rood, H. J. 1987, ApJS, 63, 555

van Weeren, R. J., Hoeft, M., Röttgering, H. J. A., et al. 2011 A\&A, 528, A38

Venturi, T., Giacintucci, S., Dallacasa, D., et al. 2008, A\&A, 484, 327 